sein, rein, glioblastome, tumeurs pédiatriques, etc.) démontrent l'intérêt suscité par les modèles de xénogreffes tumorales. De plus, les premières tentatives de corrélation entre le pronostic du patient et la réponse pharmacologique des xénogreffes correspondantes ont déjà montré quelques succès $[2,3]$.

La valeur des collections de xénogreffes dérivées de tumeurs de patients dépend essentiellement de deux paramètres importants : le standard élevé d'obtention des tumeurs issues de résections chirurgicales, et le haut niveau de caractérisation des xénotumeurs, tant sur le plan histoclinique que moléculaire ou pharmacologique. Pour atteindre ces objectifs, I'un des principaux défis demeure l'importance des ressources nécessaires, à la fois financières et matérielles. Peu d'institutions peuvent, isolément, se permettre de consacrer autant de moyens à l'établissement de ces différentes collections (plusieurs pathologies tumorales différentes) de xénotumeurs. À ce titre, l'initiative CReMEC a été un modèle de collaboration avec, notamment, la mutualisation de moyens à la fois intellectuels, financiers et matériels. De plus, si l'on en juge par l'augmentation quasi exponentielle d'initiatives similaires à travers le monde ces dernières années, le consortium a été véritablement précurseur et ce, au moment où l'intérêt du recours aux xénogreffes faisait l'objet de nombreuses discussions. Fort de ce premier succès, le consortium tente à présent d'élargir son effort à huit nouvelles pathologies, tout en intégrant de nouveaux partenaires à l'échelon national. $\diamond$

Contribution to new therapeutics against colorectal cancer:

\section{the CReMEC initiative}

\section{LIENS D'INTÉRÊT}

Une licence commerciale exclusive concernant l'ensemble des xénogreffes dérivées de patients décrites dans cet article a été accordée à la société Oncodesign par le consortium CReMEC. L'auteur déclare n'avoir aucun lien d'intérêt concernant les données publiées dans cet article.

\section{REMERCIEMENTS}

L'auteur remercie les membres du consortium CReMEC qui ont participé à l'ensemble du travail, ainsi que le Ministère français de l'industrie et le département du Val de Marne, pour leur soutien financier. L'auteur remercie également Cyril Berthet (Oncodesign) et Alain
Pierré (institut de recherches international Servier) pour leur relecture de ce manuscrit.

\section{RÉFÉRENCES}

1. Julien S, Merino-Trigo A, Lacroix L, et al. Characterization of a large panel of patient-derived tumor xenografts representing the clinical heterogeneity of human colorectal cancer. Clin Cancer Res 2012 ; 18 : 5314-28.

2. DeRose $Y S$, Wang G, Lin YC, et al. Tumor grafts derived from women with breast cancer authentically reflect tumor pathology, growth, metastasis and disease outcomes. Nat Med 2011; 17 : 1514-20.

3. Hidalgo M, Bruckheimer $\varepsilon$, Rajeshkumar NV, et al. A pilot study of treatment guided by personalized tumorgrafts in patients with advanced cancer. Mol Cancer Ther 2011; 10 : 1311-6.

4. Marangoni $\varepsilon$, Vincent-Salomon A, Laurent $C$, et al. A new model of patient tumor-derived breast cancer xenograft for preclinical assays. Clin Cancer Res $2007 ; 13$ : 3989-98.

5. Forbes SA, Bhamra G, Bamford S, et al. The catalogue of somatic mutations in cancer (COSMIC). Curr Protoc Hum Genet 2008 ; chapter 10 : Unit 10.11.

6. Bergamaschi A, Hjortland GO, Decraene C, et al. Molecular profiling and characterization of luminallike and basal-like in vivo breast cancer xenograft models. Mol Oncol $2009 ; 3: 469-82$.

7. Reyal F, Guyader D, Decraene C, et al. Molecular profiling of patient-derived breast cancer xenografts. Breast Cancer Res 2012 ; 14 : R11.

8. Ding L, Ellis MJ, LiS, et al. Genome remodelling in a basal-like breast cancer metastasis and xenograft. Nature 2010 ; 464 : 999-1005.

9. Rothenberg ML. Efficacy and toxicity of irinotecan in patients with colorectal cancer. Semin Oncol 1998 ; $25: 39-46$

10. Billaud M. L'hétérogénéité intratumorale : un obstacle darwinien à la médecine personnalisée ? Med Sci (Paris) $2012 ; 28: 1116-9$

\title{
NOUVELle
}

\section{Hyperprolactinémie et infertilité}

\section{Approche physiopathologique}

Charlotte Sonigo, Jacques Young, Nadine Binart
Inserm U693, Faculté de médecine Paris-Sud, Université Paris-Sud, 63, rue Gabriel Péri, 94276 Le Kremlin-Bicêtre, France. nadine.binart@inserm.fr
> Il est communément admis que l'allaitement empêche la survenue d'une nouvelle grossesse. Ce phénomène est secondaire à la production intense d'une hormone hypophysaire, la prolactine, pendant la grossesse et l'allaitement. Des taux circulants élevés de prolactine, ou hyperprolactinémie, sont également observés dans certaines situations pathologiques, telles que les adénomes hypophysaires à prolactine, la situation la plus fréquente.
L'hyperprolactinémie est définie par une élévation de la concentration plasmatique de prolactine au-delà de la limite supérieure des valeurs mesurées dans la population normale, celles-ci variant, selon les méthodes utilisées, de 15 à $25 \mathrm{ng} / \mathrm{ml}$. Cette élévation de la prolactine peut être responsable, chez l'homme, d'une baisse de la libido, d'infertilité ou plus rarement de galactorrhée. Chez la femme, c'est une des principales causes de galactorrhée, de troubles du cycle ou d'infertilité par anovulation. Les mécanismes moléculaires exacts du déficit gonadotrope partiel conduisant à l'infertilité étaient, jusqu'à ce jour, inconnus. Une étude récente de notre groupe a permis de mettre en évidence que la kisspeptine, un nouvel acteur de l'axe gonadotrope connu depuis les années 2000, était le chaînon manquant de la voie conduisant au déficit gonadotrope dans l'hyperprolactinémie [1]. 
Cette découverte permet, non seulement de mieux comprendre la physiopathologie de l'infertilité dans l'hyperprolactinémie, mais elle constitue également une avancée thérapeutique, notamment parce qu'elle offre une stratégie d'induction d'une ovulation chez les patientes hyperprolactinémiques résistantes ou intolérantes au traitement médical classique.

\section{Hyperprolactinémie et infertilité : les anciennes hypothèses}

Il est admis depuis plus de 30 ans que ce déficit gonadotrope partiel résulte de l'altération de la sécrétion de GnRH (gonadotropin releasing hormone), principale hormone gouvernant l'axe gonadotrope. En effet, des études réalisées dans les années 1980 ont montré que l'administration de $\mathrm{GnRH}$, chez l'homme comme chez la femme, rétablissait un fonctionnement testiculaire ou ovarien normal chez des sujets dont l'hyperprolactinémie était persistante [2]. Depuis, plusieurs hypothèses ont été formulées pour expliquer cette altération de la sécrétion de GnRH. Selon la théorie communément admise, mais controversée, le déficit gonadotrope serait secondaire à une augmentation du tonus dopaminergique qui inhiberait la pulsatilité de la sécrétion de $\mathrm{GnRH}$, mais peu de travaux confortent cette hypothèse. Selon d'autres travaux anciens, la prolactine en excès agirait directement sur les neurones à $\mathrm{GnRH}$ [3] ; pourtant, ces derniers n'expriment que peu ou pas le récepteur de la prolactine [4]. II semblerait donc exister une voie intermédiaire impliquant d'autres systèmes neuronaux.

Les kisspeptines et leurs récepteurs : nouveaux acteurs fondamentaux

\section{de l'axe gonadotrope}

Selon la représentation classique qui prévaut avant 2003, l'axe gonadotrope est constitué des neurones hypothalamiques sécrétant de manière pulsatile la GnRH ; ceux-ci stimulent les cellules gonadotropes hypophysaires dont la sécrétion des gonadotrophines LH (luteinizing hormone) et FSH (follicle- stimulating hormone) est induite. (es gonadotrophines stimulent la production des stéroïdes sexuels par l'ovaire: œstradiol et progestérone chez la femme. Ces trois niveaux de l'axe gonadotrope sont liés par une régulation complexe faisant intervenir un double rétrocontrôle, positif et négatif.

$\varepsilon n 2003$, deux équipes ont mis en évidence simultanément des mutations du récepteur de la kisspeptine (KISSIR anciennement appelé GPR54) chez des individus atteints d'un hypogonadisme hypogonadotrope avec une absence de puberté $[5,6]$. Cette découverte, la plus excitante qui ait été faite dans le domaine de la reproduction depuis celle de la GnRH dans les années 1970, a conduit à démontrer que la kisspeptine était un des déclencheurs de la puberté et le plus puissant stimulateur de la sécrétion de $\mathrm{GnRH}$. Rien ne laissait prévoir que ce peptide, codé par le gène KISSI identifié en 1996 dans la ville de Hershey en Pennsylvannie (États-Unis) (connue pour ses chocolats « kisses »), d'abord impliqué comme suppresseur de métastases dans des cellules de mélanome, serait considéré aujourd'hui comme l'effecteur fondamental de l'axe gonadotrope. Depuis 2003, un grand nombre d'études ont montré que les kisspeptines, sécrétées par des neurones hypothalamiques, étaient impliquées dans les rétrocontrôles positif et négatif des stéroïdes sexuels sur l'axe hypothalamo-hypophysaire et étaient le relais de signaux périphériques influant sur l'axe gonadotrope, comme la leptine ou la photopériode [7]. C'est dans ce contexte que l'hypothèse d'une intervention des kisspeptines dans le déficit gonadotrope secondaire à I’hyperprolactinémie a été posée.

\section{Les kisspeptines, chaînon manquant} du déficit gonadotrope dans l'hyperprolactinémie

Notre étude, publiée récemment, a validé cette hypothèse [1]. Nous avons mis au point un modèle de souris hyperprolactinémique, grâce à des micropompes délivrant de la prolac- tine de manière continue pendant quatre semaines. Rapidement, ces souris développent des altérations de la cyclicité, avec des cycles irréguliers voire inexistants, reproduisant ainsi la pathologie humaine. L'anovulation était confirmée par l'analyse histologique des ovaires qui constatait une absence de corps jaunes, témoins habituels de l'ovulation. Afin de valider l'hypothèse d'une implication de la kisspeptine, des injections régulières intrapéritonéales de kisspeptine ou de placebo ont été administrées à ces souris hyperprolactinémiques. De manière très intéressante, une restauration des cycles réguliers a été observée chez les souris recevant de la kisspeptine; elle s'accompagnait de la présence d'un nombre normal de corps jaunes dans les ovaires. Au contraire, aucune amélioration n'était constatée chez les souris traitées par placebo. La kisspeptine semblait donc restaurer une cyclicité et une ovulation normales chez les souris hyperprolactinémiques.

L'analyse de l'expression des peptides hypothalamo-hypophysaires a permis d'évaluer les mécanismes moléculaires impliqués. Les taux des hormones hypophysaires FSH et LH étaient diminués chez les animaux ayant une hyperprolactinémie, alors qu'il n'y avait pas de variation de l'expression du gène gnrh. Ces résultats sont en faveur d'une altération de la sécrétion ou de la pulsatilité de GnRH à l'origine d'une baisse de la production de LH et FSH. L'analyse de l'expression de kisspeptine dans l'hypothalamus de ces souris, ainsi que l'analyse immunohistochimique de ce peptide dans les noyaux hypothalamiques, révèlent sa nette diminution chez les souris en hyperprolactinémie par rapport aux animaux témoins.

La prolactine en excès induit donc, chez la souris comme dans l'espèce humaine, une altération de la sécrétion de GnRH. Ces données ont été confirmées par des études in vitro. En effet, des explants hypothalamiques de souris 
mis en culture en présence d'un excès de prolactine sécrétaient peu de $\mathrm{GnRH}$. Cependant, une sécrétion normale était restaurée lorsque ces explants étaient secondairement mis en culture dans un milieu contenant à la fois de la prolactine et de la kisspeptine. Ces résultats confirment que l'hyperprolactinémie induit un dysfonctionnement de la sécrétion de $\mathrm{GnRH}$, et que celle-ci est restaurée par la kisspeptine (Figure I). Une étude publiée pendant la réalisation de ce travail conforte cette hypothèse [8]. Elle montre en effet que chez le rongeur, peu de neurones à GnRH expriment l'ARNm du récepteur de la prolactine, alors que la majorité des neurones exprimant des transcrits codant pour la kisspeptine coexpriment ceux qui codent pour le récepteur de la prolactine. Ces résultats plaident donc en faveur d'une action indirecte de la prolactine sur les neurones à $\mathrm{GnRH}$, via les neurones à kisspeptines.

\section{Perspectives}

Cette étude, réalisée chez des rongeurs, a donc permis de décrypter les mécanismes induisant le déficit gonadotrope dans le cas de l'hyperprolactinémie. Afin de confirmer cette hypothèse chez la femme, une étude est en cours chez des patientes ayant une hyperprolactinémie résistante au traitement médicamenteux, et qui reçoivent des injections de kisspeptine. Si ces données sont validées, l'administration de kisspeptine pourrait entrer dans l'arsenal thérapeutique offert aux femmes ayant une hyperprolactinémie résistante au traitement médical. Cette approche serait essentiellement ciblée sur la prise en charge de leur infertilité. $\diamond$

Hyperprolactinemia and infertility: a new physiopathological approach

\section{LIENS D'INTÉRÊT}

Les auteurs déclarent n'avoir aucun lien d'intérêt concernant les données publiées dans cet article.

\section{RÉFÉRENCES}

1. Sonigo C, Bouilly J, Carré N, et al. Hyperprolactinemiainduced ovarian acyclicity is reversed by kisspeptin administration. J Clin Invest $2012 ; 122: 3791-5$.

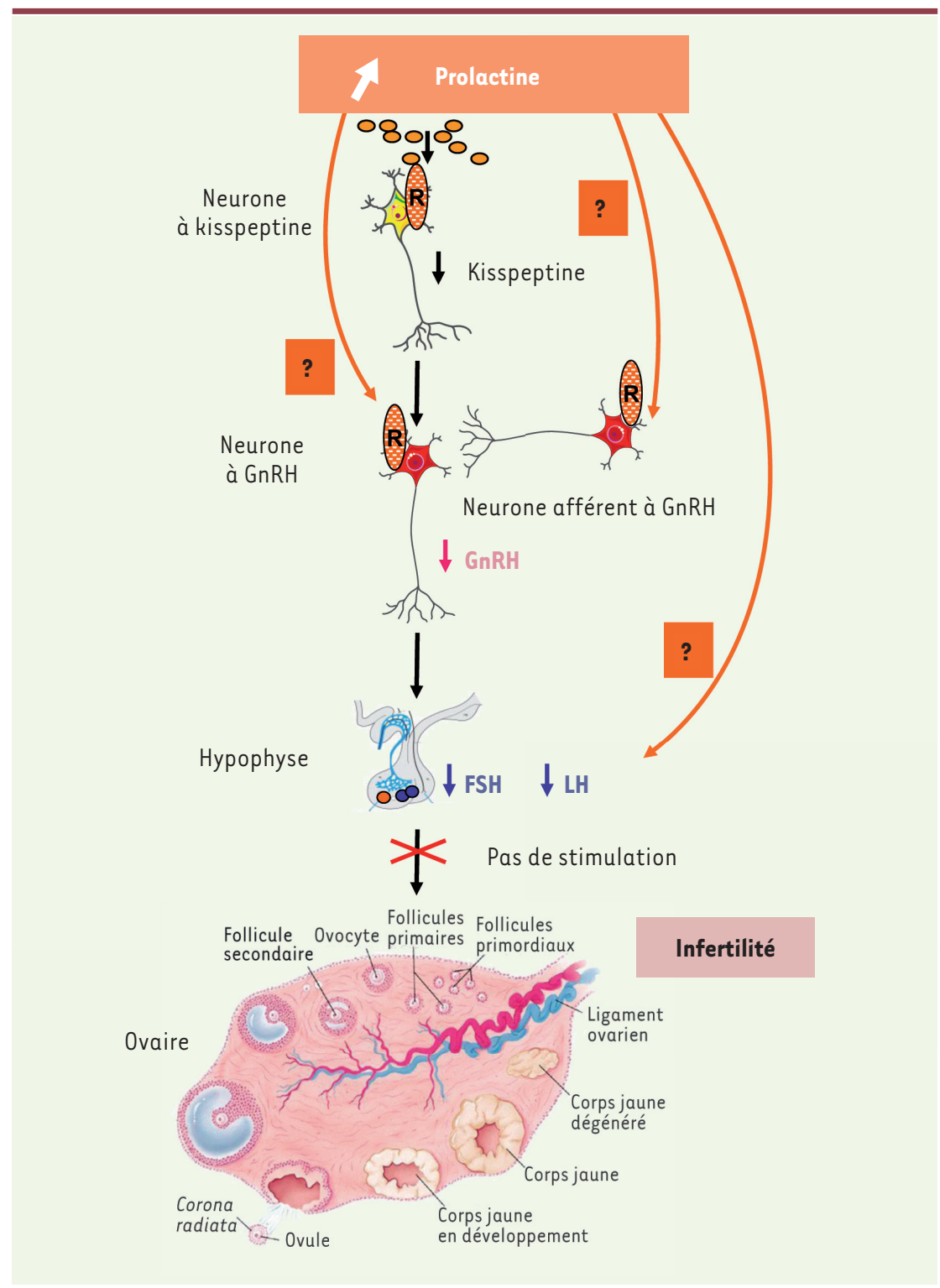

Figure 1. Mécanismes de l'hypogonadisme induit par l'hyperprolactinémie. Les taux élevés de prolactine (orange) font baisser l'expression de kisspeptine dans les neurones à kisspeptine (jaune) par le biais des récepteurs de la prolactine $(\mathrm{R})$. Ceci provoque une baisse du relargage de la $\mathrm{GnRH}$ (rouge), conduisant à une perte du signal GnRH nécessaire à l'ovulation. Les hormones hypophysaires FSH et LH (bleu) sont alors faiblement sécrétées. La prolactine pourrait aussi avoir des effets directs sur les neurones à GnRH et/ou les cellules gonadotropes, ou encore d'autres neurones à GnRH afférents.

2. Bouchard P, Lagoguey M, Brailly S, Schaison G. Gonadotropin-releasing hormone pulsatile administration restores luteinizing hormone pulsatility and normal testosterone levels in males with hyperprolactinemia. J Clin Endocrinol Metab 1985; $60: 258-62$.

3. Milenković L, D’Angelo G, Kelly PA, Weiner RI. Inhibition of gonadotropin hormone-releasing hormone release by prolactin from GTl neuronal cell lines through prolactin receptors. Proc Natl Acad Sci USA $1994 ; 91: 1244-7$

4. Grattan DR, Jasoni CL, Liu X, et al. Prolactin regulation of gonadotropin-releasing hormone neurons to suppress luteinizing hormone secretion in mice. Endocrinology 2007 ; 148 : 4344-51.
5. de Roux N, Genin E, Carel J-C, et al. Hypogonadotropic hypogonadism due to loss of function of the KiSS1derived peptide receptor GPR54. Proc Natl Acad Sci USA 2003 ; 100 : 10972-6.

6. Seminara SB, Messager S, Chatzidaki $\varepsilon$, et al. The GPR54 gene as a regulator of puberty. $N$ EnglJ Med 2003 ; 349 : 1614-27.

7. Roa J, Navarro VM, Tena-Sempere M. Kisspeptins in reproductive biology: consensus knowledge and recent developments. Biol Reprod 2011 http://www.ncbi.nlm.nih.gov/pubmed/21677307

8. Kokay IC, Petersen SL, Grattan DR. Identification of prolactin-sensitive GABA and kisspeptin neurons in regions of the rat hypothalamus involved in the control of fertility. Endocrinology 2011 ; 152 : 526-35 